Objectives The study objective was to document the division of occupations by sex/gender in Canada and to examine the trends since 1991. The evidence is intended to inform occupational health and safety policies and procedures by including issues of sex/gender as part of the discourse on risk prevention, where warranted.

Methods Data obtained from the last six Canadian Censuses of Population (1991-2016) were analyzed and descriptive statistics were used to examine the labour force composition within various resolutions of the National Occupational Classification (NOC) codes by sex/gender. GEE Poisson regression models were used to generate time- and occupation-adjusted estimates for incidence rate ratios with 95\% confidence intervals for sex/gender differences in the labour force. Highly divided occupations were defined as those with $75 \%$ or more men or women.

Results Of the 500 4-digit occupational categories representing 2,892 data points over the 25-year period, 58\% were highly divided, with more than three-quarters of these incidences being for male-dominated occupations, with less than onequarter being for female-dominated occupations. GEE analyses of these occupation groups within broad occupational groups suggested relative stability in the gendered nature of occupations over time, with a statistically significant reduction in the proportion of highly divided occupations only observed among occupations broadly grouped within natural and applied sciences.

Conclusion The Canadian workforce remains highly sexed/gendered. While the distribution of men and women within occupations is driven by complex factors, our inquiry into these found that systems of gender essentialism, organizational hierarchies that favour men, and labour markets that are changeresistant remain challenges.

\section{0-250 ASSOCIATION OF CHILD NEURODEVELOPMENTAL AND BEHAVIOURAL PROBLEMS WITH MATERNAL UNEMPLOYMENT IN A POPULATION-BASED BIRTH COHORT}

'Joana Amaro, Maja Popovic, Milena Maule, Ingrid Sivesind Mehlum, Raquel Lucas, Raquel Costa. 'Institute of Public Health, University of Porto, Portugal

\subsection{6/OEM-2021-EPI.136}

Introduction Mothers typically adjust their work participation in order to meet family needs, including their children's wellbeing. However, there is scarce evidence on the impact of children's poor mental health on maternal employment in less affluent European economies, where the dual-earner family structure is often necessary to make ends meet.

Objectives To estimate associations between suspected and diagnosed neurodevelopmental and behavioural problems in 7 year-old children and maternal unemployment at child ages 7 and 10 , in a Portuguese birth cohort.

Methods We evaluated 5754 mother-child pairs of the population-based birth cohort Generation XXI. Data on child suspected and diagnosed neurodevelopmental and behavioural problems, namely learning, attention and language problems, externalizing behaviours, developmental delay, autism spectrum disorders, and other neurodevelopmental problems, were retrieved at 7 years of age using caregivers' reports. Maternal employment status was collected at child age 7 and 10. Robust Poisson regression models were used to calculate prevalence ratios (PRs) and 95\% confidence intervals (CIs).

Results After adjustment for maternal age, education, being a single mother, singleton/multiple pregnancy, having children under the age of 6 years, household monthly income and maternal mental disorder diagnosis, women were more likely to be unemployed at child age 10 if the child had, up to age 7 , any of the following suspected problems: externalizing behaviours ( $\mathrm{PR}=1.20$, 95\% CI [1.03-1.40]), developmental delay $(\mathrm{PR}=1.46$, 95\% CI [1.12-1.91]), or an autism spectrum disorder $(\mathrm{PR}=1.62,95 \% \mathrm{CI}$ [0.99-2.65]). When the exposure was restricted to clinically diagnosed disorders, the magnitude of associations remained similar, although estimates were less precise. Associations with unemployment were stronger when the child was $10 \mathrm{com}$ pared to when the child was 7 .

Conclusion Having a child with a developmental or behavioural problem or an autism spectrum disorder at age 7 was associated with maternal unemployment three years later, placing families at a higher risk of financial vulnerability.

\section{0-378 THE ROLE OF GENDER IN THE ASSOCIATION BETWEEN SEXUAL HARASSMENT AT WORK AND SUICIDAL IDEATION: A STUDY WITH CIVIL SERVANTS FROM A MIDDLE-INCOME COUNTRY}

${ }^{1}$ Fernando Feijó, Inaê Dutra Valério, Débora Gräf, Maria del Pilar Flores-Quispe, Anaclaudia Fassa. 'Federal University of Bahia, Brazil

\subsection{6/OEM-2021-EPI.137}

Introduction Sexual harassment is associated with several mental health problems, including suicidality. Despite its terrible consequences for individuals, it is frequently an invisible occupational risk. Women are more likely to be harassed than men. However, little is known about gender differences and effect modification on this association.

Objectives To analyse the association between sexual harassment at work and suicidal ideation(SI) in civil servants from a state in Southern Brazil, investigating gender differences and interaction.

Methods This is a cross-sectional study with data from a web survey with employees from the Federal Judicial Court in Southern Brazil. Sexual harassment was evaluated using a structured questionnaire with specific questions about harassment and violence. SI was assessed using the Self-Reporting Questionnaire (SRQ-20). Poisson regression was used to test associations of interest and estimate prevalence ratios (PR). Stratified analyses by sex were performed.

Results 1,636 workers provided complete information. The prevalence of self-reported sexual harassment was 5.6\%, $2.7 \%$ among males and $8.2 \%$ among females. The prevalence of SI was $4.2 \%, 5.0 \%$ in males and $3.5 \%$ in females. Among harassed men, the prevalence of SI was $27.3 \%$. Sexual harassment was positively associated with SI after adjustment for age and educational level(PR 2.31; 95\%CI 1.134.72). In the analysis stratified by sex, the association remained positive only in males $(\mathrm{PR}=4.70$; 95\%CI 1.90 11.66), after adjustment for age, educational level and personality traits.

Conclusions Gender play an important role in the association between sexual harassment and suicide. Approaching the understanding of gender to the measured variable of sex, the 